\title{
Drug-induced Sleep Endoscopy: Are there Predictors for Failure of Oral Appliance Treatment?
}

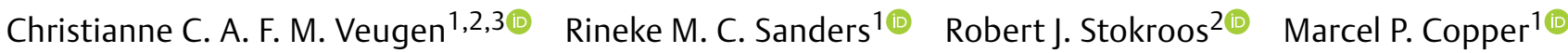 \\ ${ }^{1}$ Department of Otorhinolaryngology, Head and Neck Surgery, Sint \\ Address for correspondence Christianne C. A. F. M. Veugen, MD, \\ Antonius Hospital, Nieuwegein, The Netherlands \\ 2 Department of Otorhinolaryngology, Head and Neck Surgery, \\ University Medical Center Utrecht, Utrecht, The Netherlands \\ Department of Otorhinolaryngology, Head and Neck Surgery, \\ University Medical Center Groningen, Hanzeplein 1, 9713 GZ \\ Groningen, The Netherlands (e-mail: c.c.a.f.m.veugen@umcg.nl).
}

3 Department of Otorhinolaryngology, Head and Neck Surgery,

University Medical Center Groningen, Groningen, The Netherlands

Int Arch Otorhinolaryngol 2022;26(3):e339-e347.

Abstract
Keywords
- obstructive sleep
apnea
- drug-induced sleep
endoscopy
- oral appliance
treatment
- mandibular
advancement device
- treatment outcome
prediction

Introduction In the literature, evidence is lacking on the predictive value of druginduced sleep endoscopy (DISE) for oral appliance treatment (OAT).

Objectives The aim of the present study is to evaluate whether DISE with concomitant mandibular advancement maneuver can predict failure of OAT.

Methods An observational retrospective study including patients diagnosed with obstructive sleep apnea (OSA) who previously received OAT. Results of DISE were analyzed in a group with documented OAT failure (apnea-hypopnea index [AHI] $>10$ events/hour or $<50 \%$ reduction) and a group with OAT benefit (AHI $<10$ events/hour or $>50 \%$ reduction). The upper airway was assessed using the velum, oropharynx, tongue base, epiglottis (VOTE) classification. Additionally, a mandibular advancement maneuver, manually protruding the mandible by performing a jaw thrust, was performed to mimic the effect of OAT.

Results The present study included 50 patients with OAT failure and 20 patients with OAT benefit. A subgroup analysis of patients with OAT failure and an $\mathrm{AHI}<30$ events/hour included 26 patients. In the OAT failure group, $74 \%$ had a negative jaw thrust maneuver. In the subgroup with an $\mathrm{AHI}<30$ events/hour, $76.9 \%$ had a negative jaw thrust maneuver. In the OAT benefit group, $25 \%$ had a negative jaw thrust maneuver $(p<0.001)$.

Conclusions A negative jaw thrust maneuver during DISE can be a valuable predictor for OAT failure, independent of AHI. Drug-induced sleep endoscopy should be considered as a diagnostic evaluation tool before starting OAT.

\section{Introduction}

Obstructive sleep apnea (OSA) is a sleep-related breathing disorder characterized by repetitive partial or complete upper airway obstruction that often results in decreased arterial oxygen saturation and arousal from sleep. ${ }^{1-4}$ The current gold standard treatment of moderate to severe OSA is continuous positive airway pressure (CPAP). ${ }^{5,6}$ However, compliance and long-term use of CPAP is rather low. ${ }^{7}$ In patients with mild to moderate OSA or in cases of CPAP received

July 12,2020

accepted after revision

March 19, 2021

published online

October 26, 2021
DOI https://doi.org/ 10.1055/s-0041-1731368. ISSN 1809-9777.

\footnotetext{
(c) 2021. Fundação Otorrinolaringologia. All rights reserved. This is an open access article published by Thieme under the terms of the Creative Commons Attribution-NonDerivative-NonCommercial-License, permitting copying and reproduction so long as the original work is given appropriate credit. Contents may not be used for commercial purposes, or adapted, remixed, transformed or built upon. (https://creativecommons.org/ licenses/by-nc-nd/4.0/) Thieme Revinter Publicações Ltda., Rua do Matoso 170, Rio de Janeiro, RJ, CEP 20270-135, Brazil
} 
intolerance, other treatment options include oral appliance treatment (OAT), a noninvasive alternative to CPAP. ${ }^{2,3,6}$ Mandibular advancement devices (MADs), which are used intraorally at night to advance the mandible, are the most common class of oral appliances. ${ }^{6}$ Oral appliance treatment appears to have higher compliance rate and a higher patient preference, with fewer side effects and greater satisfaction when compared with CPAP therapy. ${ }^{8}$ However, OAT is not always as effective in treating OSA. In a recent review article, approximately one-third of patients did not experience a therapeutic benefit. ${ }^{9}$ Finding predictors to select suitable patients that may benefit from OAT is therefore of great importance. Various anthropometric and polysomnographic predictors for OAT have been described in the literature, including lower apnea-hypopnea index (AHI), lower bodymass index (BMI), lower age, female gender, and supinedependent OSA. ${ }^{10}$ However, no diagnostic prediction tool for the effectiveness of OAT has been identified so far.

Drug-induced sleep endoscopy (DISE), first described in 1991 by Croft et al., is a diagnostic evaluation tool for the degree, level(s), and pattern of upper airway obstruction in OSA patients. ${ }^{2,11}$ During DISE, a mandibular advancement maneuver is performed as a prediction tool for the effectiveness of OAT. However, opinions concerning the performance of a mandibular advancement maneuver during DISE vary among studies, and evidence on the positive and negative predictive values are limited so far., ${ }^{3,6,12-18}$ Presently, patients are often prescribed OAT without evaluation of the upper airway through DISE. In case of ineffectiveness of OAT, there is a large delay in the appropriate treatment of the disorder and a waste of healthcare supplies.

In the present retrospective study, the DISE results from patients with documented OAT benefit and OAT failure will be analyzed, and individual predictors for OAT failure will be identified. To the best of our knowledge, this is the first study to compare DISE results both of patients with OAT failure and with OAT benefit.

\section{Materials and Methods}

\section{Study Design and Patient Population}

Data from 201 patients who were referred to this tertiary referral sleep center in the Netherlands between January 2017 and June 2019 were retrospectively analyzed. Patients referred to this center have repeatedly failed different therapies, and often present with CPAP- and OAT- failure or intolerance. Drug-induced sleep endoscopy is performed in all patients in order to consider other treatment options, such as surgical procedures and upper airway stimulation. The inclusion criteria were patients $\geq 18$ years old, previous treatment with OAT (specifically MAD) and DISE with concomitant mandibular advancement maneuver performed in this hospital. A recent apnea-hypopnea index (AHI) measured by polysomnography (PSG) or respiratory polygraphy (PG or home sleep apnea test) had to be available. The exclusion criteria were patients with no history of OAT treatment, or OAT treatment different from a MAD, missing apnea-hypopnea index (AHI), or technically inadequate $\mathrm{P}(\mathrm{S})$
$\mathrm{G}$, and if DISE was not performed in this hospital, or if a mandibular advancement maneuver was not performed. In the outpatient clinic, routine ear, nose, and throat (ENT) examination was performed. The following clinical parameters were collected for all patients: gender, age, height, weight, BMI, tonsil size (0-4), and Mallampati score. ${ }^{1-4}$

\section{Pretreatment Sleep Study}

All patients were diagnosed with OSA, which was either confirmed by PSG or respiratory PG. The variables collected were $\mathrm{AHI}$, oxygen desaturation index $\geq 3 \%$, and oxygen desaturation index $\geq 4 \%$, if available. Apnea was defined as a decrease of at least $90 \%$ of airflow from baseline for $>10$ seconds. Hypopnea was defined as a decrease of at least $30 \%$ of airflow from baseline for $>10$ seconds, associated with either an arousal or with $\geq 3 \%$ arterial oxygen saturation decrease. The mean number of apneas and hypopneas per hour of sleep (AHI) was calculated. The ODI $\geq 3 \%$ was defined as the mean number of arterial oxygen desaturations $\geq 3 \%$. The ODI $\geq 4 \%$ was defined as the mean number of arterial oxygen desaturations $\geq 4 \%$. The variables from the most recent sleep study were used in the analysis. If surgery was performed (for example, upper airway stimulation, pharyngoplasty), the last sleep study before surgery was used.

\section{Drug-induced Sleep Endoscopy}

Drug-induced sleep endoscopy was performed in a quiet operating room with dimmed lights. All procedures were performed by the same experienced ENT-surgeon (Copper, MP) with an anesthesiologist to manage sedation. Sleep was induced by an initial bolus of $1 \mathrm{mg} / \mathrm{kg}$ propofol, followed by a titration of propofol. The optimal depth of sedation was reached when the patient began to snore and/or hyporesponsiveness to vocal and tactile stimuli was achieved (Ramsay sedation level 5). Once a proper level of sedation was achieved, the upper airway was thoroughly observed by flexible fiberoptic laryngoscopy. The upper airway was assessed in the supine position using the velum, oropharynx, tongue base, epiglottis (VOTE) classification system as described by Kezirian et al. in 2011. ${ }^{19}$ Upper airway collapse was evaluated on four different levels and structures, namely the velum $(\mathrm{V})$, the oropharynx $(\mathrm{O})$, the tongue base $(\mathrm{T})$, and the epiglottis (E). The degree of obstruction was defined as 0: no obstruction (collapse $<50 \%$ ); 1 : partial collapse (between $50 \%$ and $75 \%$, typically with vibration); or 2 : complete collapse ( $>75 \%$ ). The configuration of obstruction can be classified as anteroposterior (AP), lateral (La) or concentric (Co). ${ }^{2,19}$ After the first assessment of the upper airway using the VOTE classification system, a mandibular advancement maneuver, manually protruding the mandible by performing a jaw thrust, was performed to mimic the effect of OAT. The hands of the practitioner were placed behind the angles of the mandible and thrust forward. The jaw thrust maneuver was performed without extensive force, bringing the lower incisors past the upper incisors by a couple of millimeters, producing a mild anterior protrusion of the mandible of $\sim$ $75 \%$ of the maximal protrural range. The jaw thrust 
maneuver was called positive if the obstruction was discontinued on all levels. The jaw thrust maneuver was called negative if the obstruction was still present on one or more levels.

\section{Data Analysis}

Our primary analysis describes the patient group with documented OAT failure. Oral appliance treatment failure was defined as an insignificant decrease in AHI on a followup sleep study (AHI $>10$ events/hour or $<50 \%$ reduction from the baseline AHI). Oral appliance treatment intolerance, like temporomandibular dysfunction, dental pain or hypersalivation, was not counted as OAT failure. The secondary analysis describes the patient group with documented OAT benefit. Oral appliance treatment benefit was defined as a significant decrease in $\mathrm{AHI}$ on a follow-up sleep study (AHI $<10$ events/hour or $>50 \%$ reduction from baseline AHI). One subgroup analysis was performed in the patient group with OAT failure. This subgroup analysis describes the patient group with documented OAT failure and an $\mathrm{AHI}<30$ events/hour. This cutoff point was used to obtain comparable baseline characteristics. Furthermore, the Dutch guideline regarding OSA treatment states that OAT is not the first treatment choice in patients with an $\mathrm{AHI}>30$ events/h.

\section{Statistical Analysis}

The statistical analysis was performed by using IBM SPSS Statistics for Windows version 24 (IBM Corp., Armonk, NY, USA). Continuous data are presented as means with standard deviations (SDs). Categorical variables are presented as frequencies with percentages. Comparisons between groups were performed using chi-squared tests for categorical variables and the unpaired Student $t$ test for continuous variables. The predictive performance of the jaw thrust maneuver for OAT failure was estimated from the area under the curve (AUC) obtained by receiver operator characteristic (ROC) curves. Additionally, sensitivity, specificity, positive predictive value (PPV), and negative predictive value (NPV) were calculated using four-grid contingency tables. All estimates are reported with their respective $95 \%$ confidence interval (CI). The association between various individual demographic data and clinical variables obtained from the sleep study test and DISE and the presence of OAT failure was established by using a multivariate logistic regression model (backward stepwise selection, $p<0.05)$. All variables that were associated with OAT failure $(p<0.20)$ were entered into the regression model. Additionally, a multivariate logistic regression analysis adjusted for confounding factors was used to assess the relation between OAT failure and the jaw thrust maneuver. A twotailed $p$-value $<0.05$ was considered statistically significant.

\section{Results}

\section{Baseline Characteristics}

Seventy patients met our inclusion criteria. The patients were subdivided in an OAT failure and an OAT benefit group; 50 patients with OAT failure were included in the primary analysis and 20 patients with OAT benefit were included in

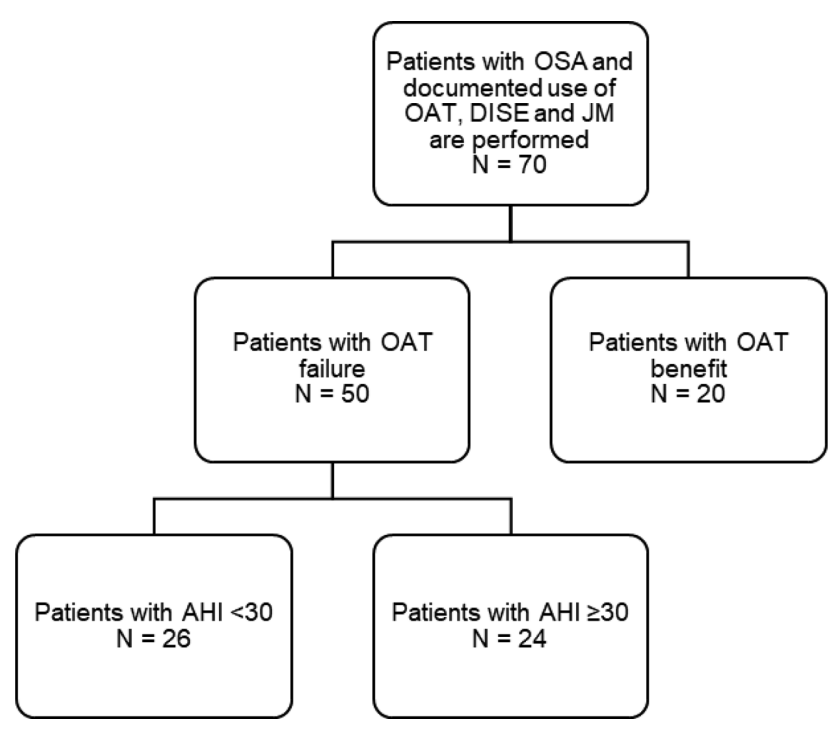

Fig. 1 Flowchart of patient inclusion. $\mathrm{AHI}=$ apnea-hypopnea index. DISE $=$ drug-induced sleep endoscopy. JM = jaw thrust maneuver. $\mathrm{OAT}=$ oral appliance treatment. OSA = obstructive sleep apnea.

the secondary analysis. The subgroup analysis of patients with OAT failure and an $\mathrm{AHI}<30$ events/hour included 26 patients (-Fig. 1 ).

\section{Primary Analysis - OAT Failure $(n=50)$}

Baseline characteristics are shown in -Table 1. Sleep study data was obtained by PSG in 68\% (34/50) of the patients and by PG in $32 \%$ (16/50) of the patients. A total of $84 \%(42 / 50)$ of the patients with OAT failure were male. The mean age was $57.2 \pm 10.8$ years old, with a mean BMI of $28.0 \pm 2.8 \mathrm{~kg} / \mathrm{m}^{2}$, and a mean AHI of $31.1 \pm 17.1$ events/hour. The mean ODI $\geq$ $3 \%$ was $30.6 \pm 16.8$ events/hour, and the mean ODI $\geq 4 \%$ was $20.0 \pm 15.2$ events/hour. Previous tonsillectomy was performed in $36 \%$ (18/50) of the patients. The distribution of the levels and the pattern of upper airway collapse during DISE is shown in - Table 2. A total of 74\% (37/50) of the patients with OAT failure had a negative jaw thrust maneuver (Tab. 1, -Fig. 2a).

\section{Secondary Analysis - OAT Benefit $(n=20)$}

Baseline characteristics are shown in -Table 1. Sleep study data was obtained by PSG in 90\% (18/20) of the patients and by PG in $10 \%(2 / 20)$ of the patients. A total of $70 \%(14 / 20)$ of the patients with OAT benefit was male. The mean age was $55.6 \pm 7.6$ years old, with a mean BMI of $26.8 \pm 2.9 \mathrm{~kg} / \mathrm{m}^{2}$, and a mean AHI of $22.8 \pm 10.4$ events/hour. The mean ODI $\geq 3 \%$ was $18.7 \pm 10.2$ events/hour, and the mean ODI $\geq 4 \%$ was $12.1 \pm 8.8$ events/hour. Previous tonsillectomy was performed in $70 \%(14 / 20)$ of the patients. The distribution of the levels and the pattern of upper airway collapse during DISE is shown in - Table 2. A total of 25\% (5/20) of the patients with OAT benefit had a negative jaw thrust maneuver (Tab. 1, - Fig. 2b).

Sleep study data was obtained by PSG in $90 \%$ of the patients with OAT benefit and in $68 \%$ of the patients with OAT failure. This difference was statistically significant 
Table 1 Baseline characteristics

\begin{tabular}{|c|c|c|c|c|c|c|}
\hline $\begin{array}{l}\text { Baseline } \\
\text { characteristics }\end{array}$ & & $\begin{array}{l}\text { Patients with OAT } \\
\text { failure. }(n=50)\end{array}$ & $\begin{array}{l}\text { Patients with OAT } \\
\text { benefit. }(n=20)\end{array}$ & $\begin{array}{l}\text { Significance } \\
\text { (p-value })^{* * *}\end{array}$ & $\begin{array}{l}\text { Patients with } \\
\text { OAT failure } \\
\text { and AHI }<30 \text {. } \\
(n=26)\end{array}$ & $\begin{array}{l}\text { Significance } \\
(p \text {-value })^{* * * *}\end{array}$ \\
\hline & & \multicolumn{5}{|l|}{ Number (\%) } \\
\hline \multirow[t]{2}{*}{ Male patients } & & $42(84)$ & $14(70)$ & 0.202 & $22(84.6)$ & 0.292 \\
\hline & & \multicolumn{5}{|l|}{ Mean \pm SD } \\
\hline Age in years & & $57.2 \pm 10.8$ & $55.6 \pm 7.6$ & 0.530 & $54.6 \pm 11.1$ & 0.739 \\
\hline BMI & & $28.0 \pm 2.8$ & $26.8 \pm 2.9$ & 0.103 & $27.6 \pm 2.8$ & 0.353 \\
\hline $\mathrm{AHI}$ & & $31.1 \pm 17.1$ & $22.8 \pm 10.4$ & 0.017 & $18.2 \pm 6.4$ & 0.069 \\
\hline $\mathrm{ODI} \geq 3 \%$ & & $30.6 \pm 16.8$ & $18.7 \pm 10.2$ & 0.006 & $20.8 \pm 9.0$ & 0.487 \\
\hline \multirow[t]{2}{*}{$\mathrm{ODI} \geq 4 \%$} & & $20.0 \pm 15.2$ & $12.1 \pm 8.8$ & 0.048 & $13.2 \pm 7.8$ & 0.704 \\
\hline & & \multicolumn{5}{|l|}{ Number (\%) } \\
\hline \multirow[t]{5}{*}{ Tonsil size } & 0 & $18(36)$ & $14(70)$ & \multirow[t]{5}{*}{0.003} & $11(42.3)$ & \multirow[t]{5}{*}{$0.285^{*}$} \\
\hline & 1 & $24(48)$ & $1(5)$ & & $12(46.2)$ & \\
\hline & 2 & $8(16)$ & $5(25)$ & & $3(11.5)$ & \\
\hline & 3 & $0(0)$ & $0(0)$ & & $0(0)$ & \\
\hline & 4 & $0(0)$ & $0(0)$ & & $0(0)$ & \\
\hline \multirow[t]{4}{*}{ Mallampati score ${ }^{* *}$} & 1 & $4(8)$ & $1(5.3)$ & \multirow[t]{4}{*}{0.827} & $3(11.5)$ & \multirow[t]{4}{*}{$0.392^{*}$} \\
\hline & 2 & $15(30)$ & $6(31.6)$ & & $9(34.6)$ & \\
\hline & 3 & $11(22)$ & $4(21.1)$ & & $6(23.1)$ & \\
\hline & 4 & $20(40)$ & $8(42.1)$ & & $8(30.8)$ & \\
\hline \multicolumn{7}{|l|}{$\begin{array}{l}\text { Degree of } \\
\text { obstruction } \\
\text { according to the } \\
\text { VOTE classification } \\
(0-2) \text { : }\end{array}$} \\
\hline Velum & & \multirow{4}{*}{\multicolumn{2}{|c|}{ See -Table 2}} & $0.258^{*}$ & \multirow[t]{4}{*}{ See -Table 2} & $0.520^{*}$ \\
\hline Oropharynx & & & & $0.131^{*}$ & & $0.071^{*}$ \\
\hline Tonguebase & & & & 0.809 & & $0.611^{*}$ \\
\hline \multirow[t]{2}{*}{ Epiglottis } & & & & $0.882^{*}$ & & $0.444^{*}$ \\
\hline & & \multicolumn{5}{|l|}{ Number (\%) } \\
\hline Negative jaw thrust maneuver & & $37(74)$ & $5(25)$ & $<0.001$ & & $<0.001$ \\
\hline
\end{tabular}

Abbreviations: AHI, apnoea-hypopnoea index; BMI, body mass index; OAT, oral appliance treatment; ODI, oxygen desaturation index; SD, standard deviation.

*Mann-Whitney U test.

${ }^{* *} 1$ missing in OAT benefit group.

${ }^{* * *} p$-value primary analysis (OAT failure vs OAT benefit).

${ }^{* * * *} p$-value subgroup analysis (OAT failure $\mathrm{AHI}<30$ versus OAT benefit).

$(p=0.01)$. The group with OAT benefit contained fewer male patients and had a lower average BMI than the group with OAT failure; however, these differences were not significant $(p=0.202 ; p=0.103$, respectively). The AHI, ODI $\geq 3 \%$ and ODI $\geq 4 \%$ were significantly lower in the group with OAT benefit ( $p=0.017 ; p=0.006 ; p=0.048$, respectively). Additionally, the tonsil size was significantly lower in the group with OAT benefit ( $p=0.003$ ). The percentage of negative jaw thrust maneuver in the OAT benefit group was significantly lower than in the OAT failure group $(p<0.001)$.
Subgroup Analysis - OAT Failure $(\mathbf{A H I}<30)(n=26)$

Baseline characteristics are shown in -Table 1. A total of $84.6 \%(22 / 26)$ of the patients with OAT failure and AHI $<30$ events/hour were male. The mean age was $54.6 \pm 11.1$ years old, with a mean BMI of $27.6 \pm 2.8 \mathrm{~kg} / \mathrm{m}^{2}$, and a mean AHI of $18.2 \pm 6.4$ events/hour. The mean ODI $\geq 3 \%$ was $20.8 \pm 9.0$ events/hour, and the mean ODI $\geq 4 \%$ was $13.2 \pm 7.8$ events/hour. The distribution of the levels and the pattern of upper airway collapse during DISE is shown in - Table 2. A total of $76.9 \%(20 / 26)$ of the patients with OAT failure and 
Table 2 Overview of the distribution of the levels and pattern of upper airway collapse during DISE according to the VOTE classification

\begin{tabular}{|c|c|c|c|c|c|c|c|c|c|}
\hline \multirow[t]{3}{*}{ Level } & \multicolumn{9}{|l|}{ Direction } \\
\hline & \multicolumn{3}{|c|}{ Anteroposterior } & \multicolumn{3}{|l|}{ Lateral } & \multicolumn{3}{|c|}{ Concentric } \\
\hline & None & Partial & Complete & None & Partial & Complete & None & Partial & Complete \\
\hline \multicolumn{10}{|c|}{ Patients with OAT failure $(N=50)$} \\
\hline Velum & $0(0 \%)$ & $3(6 \%)$ & $35(70 \%)$ & - & - & - & - & - & $12(24 \%)$ \\
\hline Oropharynx & & & & $36(72 \%)$ & $12(24 \%)$ & $2(4 \%)$ & & & \\
\hline Tongue base & $8(16 \%)$ & $19(38 \%)$ & $23(46 \%)$ & & & & & & \\
\hline Epiglottis & $8(16 \%)$ & $16(32 \%)$ & $23(46 \%)$ & - & $2(4 \%)$ & $1(2 \%)$ & & & \\
\hline \multicolumn{10}{|c|}{ Patients with OAT benefit $(N=20)$} \\
\hline Velum & $0(0 \%)$ & $4(20 \%)$ & $12(60 \%)$ & - & - & - & - & - & $4(20 \%)$ \\
\hline Oropharynx & & & & $18(90 \%)$ & $1(5 \%)$ & $1(5 \%)$ & & & \\
\hline Tongue base & $2(10 \%)$ & $8(40 \%)$ & $10(50 \%)$ & & & & & & \\
\hline Epiglottis & $2(10 \%)$ & $7(35 \%)$ & $11(55 \%)$ & - & $0(0 \%)$ & $0(0 \%)$ & & & \\
\hline \multicolumn{10}{|c|}{ Patients with OAT failure and $\mathrm{AHI}<30(N=26)$} \\
\hline Velum & $0(0 \%)$ & $2(7.7 \%)$ & $19(73.1 \%)$ & - & - & - & - & - & $5(19.2 \%)$ \\
\hline Oropharynx & & & & $17(65.4 \%)$ & $8(30.8 \%)$ & $1(3.8 \%)$ & & & \\
\hline Tongue base & $5(19.2 \%)$ & $9(34.6 \%)$ & $12(46.2 \%)$ & & & & & & \\
\hline Epiglottis & $5(19.2 \%)$ & $9(34.6 \%)$ & $12(46.2 \%)$ & - & $0(0 \%)$ & $0(0 \%)$ & & & \\
\hline
\end{tabular}

Abbreviation: OAT, oral appliance treatment.

$\mathrm{AHI}<30$ had a negative jaw thrust maneuver

(Tab. 1, - Fig. 2c).

The group with OAT failure and an AHI $<30$ events/hour and the group with OAT benefit presented no significant differences in the baseline characteristics. The AHI in the OAT failure $(\mathrm{AHI}<30$ ) group was lower than the AHI in the OAT benefit group; however, this difference was not significant $(p=0.069)$. The percentage of negative jaw thrust maneuver in the OAT failure $(\mathrm{AHI}<30)$ group was significantly higher than in the OAT benefit group $(p<0.001)$ (Tab. 1).

\section{Prediction of Treatment Outcome}

In the present patient cohort, the percentage of patients with a negative jaw thrust maneuver was significantly higher in the OAT failure group $(p<0.001)$. The AHI, ODI $\geq 3 \%$, ODI $\geq$ $4 \%$ and tonsil size were also significantly higher in the OAT failure group $(p=0.017 ; p=0.006 ; p=0.048 ; p=0.003$, respectively). Multivariate logistic regression analyses were performed to establish the association between individual demographic and clinical variables and the effectiveness of OAT. Adjusting for confounding factors like previous tonsillectomy, a negative jaw thrust maneuver and a higher ODI $\geq$ $3 \%$ proved to be the strongest predictors in the OAT failure group ( $p=0.003 ; p=0.029$, respectively). Tonsil size did not prove to be a strong individual predictor in this group $(p=0.364)$. In the subgroup analysis of patients with OAT failure and $\mathrm{AHI}<30$ events/hour, only negative jaw thrust maneuver proved to be a strong predictor $(p=0.001)$. The ROC curve in - Fig. 3 a shows the discrimination of the jaw thrust maneuver between OAT failure and OAT benefit and has an AUC of 0.754 (95\%CI: 0.614-0.876). The test sensitivity of the jaw thrust maneuver is 0.75 (95\%CI: 0.53-0.89), and the test specificity is 0.74 (95\%CI: $0.60-0.84)$. The PPV is 0.54 (95\%CI: $0.36-0.70)$, and the NPV is 0.88 (95\%CI: 0.75-0.95). The ROC curve in - Fig. $\mathbf{3 b}$ shows the discrimination of the jaw thrust maneuver between OAT failure $(\mathrm{AHI}<30$ events/hour) and OAT benefit, and has an AUC of 0.760 (95\%CI: 0.614-0.905) (-Fig. 3). The test sensitivity of the

\section{A. Jaw thrust in patients with OAT- failure}

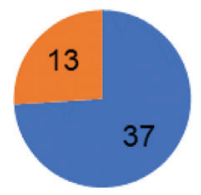

\section{B. Jaw thrust in patients without OAT- benefit}

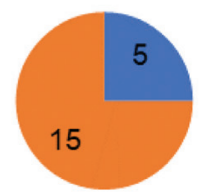

\section{Jaw thrust in patients with OAT- failure and $\mathrm{AHI}<30$}

Fig. 2 Outcome of the jaw thrust maneuver in all the patients with OAT failure (A), in patients with OAT benefit (B) and in patients with OAT failure and $\mathrm{AHI}<30$ (C).

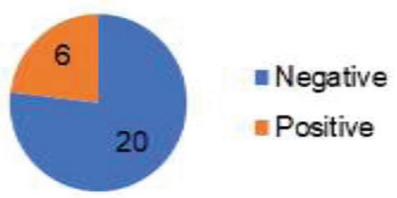




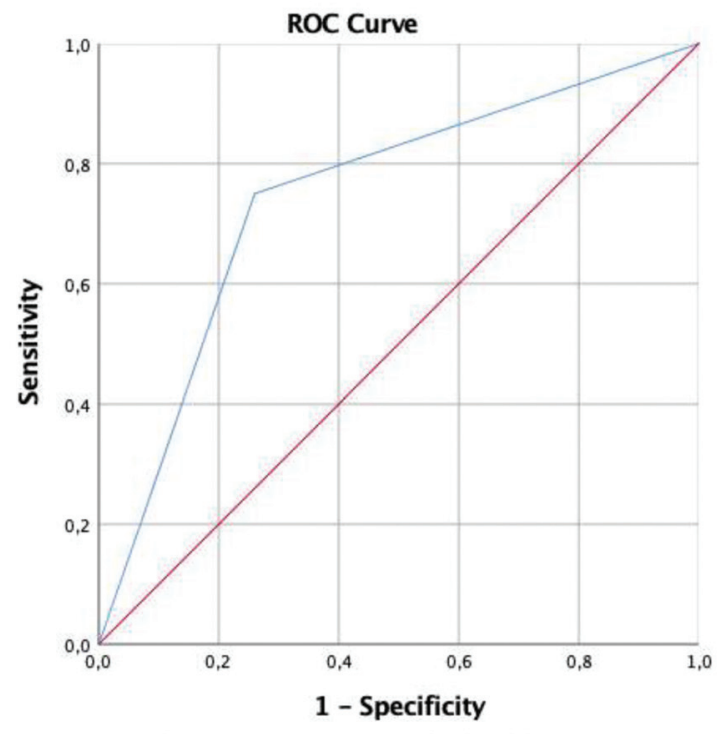

A.

Diagonal segments are produced by ties.

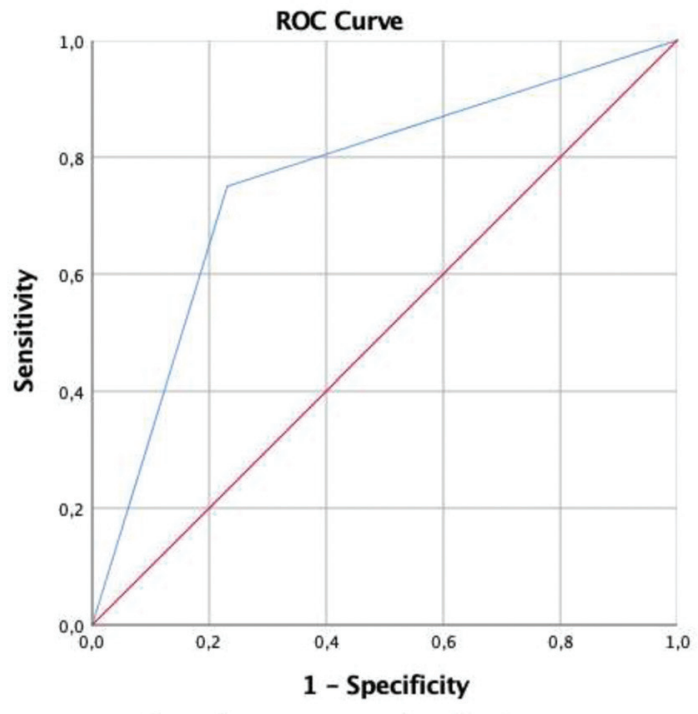

B.

Diagonal segments are produced by ties.

Fig. 3 Receiver operating characteristic (ROC) curve. A. OAT failure versus OAT benefit. The AUC is 0.754 (95\%CI: 0.614-0.876). B. OAT failure $(\mathrm{AHI}<30)$ versus OAT benefit. The AUC is 0.760 (95\% Cl: $0.614-0.905)$.

jaw thrust maneuver is 0.75 (95\%CI: 0.53-0.89), and the test specificity is 0.77 (95\%Cl: $0.58-0.89)$. The PPV is 0.71 (95\% CI: $0.5-0.86)$, and the NPV is 0.80 (95\%CI: 0.61-0.91).

\section{Discussion}

The percentage of patients with a negative jaw thrust maneuver was significantly higher in the group with OAT failure in comparison with the group with OAT benefit. The AHI, ODI $\geq 3 \%, \mathrm{ODI} \geq 4 \%$ and tonsil size were also significantly higher in the patient group with OAT failure. In a recent study by Marklund et al., it was already described that a lower AHI is a predictor for benefit from OAT. ${ }^{10}$ It could be argued that the results that we found are due to differences in $\mathrm{AHI}$ in the baseline characteristics of both patient groups, rather than to differences in outcome of the jaw thrust maneuver. To rule out this possible confounding bias in the analysis, a subgroup analysis was performed in patients with OAT failure and an $\mathrm{AHI}<30$ events/hour. In this subgroup analysis, there were no significant differences in the baseline characteristics. The percentage of patients with a negative jaw thrust maneuver was found to be significantly higher in the patients with OAT failure (AHI $<30$ events/hour). Additionally, multivariate logistic regression analyses adjusted for confounding factors were performed to assess the relation between OAT failure and the jaw thrust maneuver. The jaw thrust maneuver proved to be the strongest predictor for OAT failure.

It must be acknowledged that $25 \%$ of the patients with OAT benefit had a negative jaw thrust maneuver. When only using the results of the jaw thrust maneuver to predict OAT failure, certain patients would not receive OAT although they would benefit from the therapy. The patients with OAT benefit and a negative jaw thrust maneuver had a lower BMI and a lower AHI in comparison with the patients with OAT benefit and a positive jaw thrust maneuver. However, these differences were not significant. These results are in line with those of previous studies, indicating that lower AHI and lower BMI are also important predictors for the success of OAT. ${ }^{10}$

A total of 26\% (13/50) of the patients with OAT failure had a positive jaw thrust maneuver. These patients were older and had a higher $\mathrm{AHI}$ in comparison with the patients with a negative jaw thrust maneuver. Again, these differences were not significant. Previously, Marklund et al. already described a higher AHI and older age to be predictors for OAT failure. ${ }^{10}$ These results suggest that DISE with concomitant jaw thrust maneuver should be used together with anthropometric and polysomnographic predictors to accurately predict the success of OAT. Further prospective research needs to be done to develop a screening instrument for the effectiveness of OAT.

Seventy percent of the patients in the OAT benefit group had undergone a previous tonsillectomy, in contrast with $36 \%$ in the OAT failure group $(p=0.003$; - Table 1$)$. In - Table 2, it is shown that, in the OAT failure group, lateral collapse at the oropharyngeal level (28\%) was more common than in the OAT benefit group (10\%). These results might indicate that previous tonsillectomy is a predictor for the success of OAT. This is in line with a previous study by Op de Beeck et al., who found that a complete lateral collapse at the oropharyngeal level is related to OAT failure. ${ }^{20}$ However, a logistic regression analysis was performed, and tonsil size did not prove to be a strong individual predictor in this patient cohort. Adjusting for previous tonsillectomy, the jaw thrust maneuver proved to be a significant independent predictor.

Sleep study data was obtained by PSG from $68 \%$ of the patients with OAT failure and from $90 \%$ of the patients with OAT benefit. This difference was statistically significant $(p=0.01)$. Previous studies have shown that the AHI is underestimated in PG. ${ }^{21,22}$ If we take this into account, the mean AHI in the OAT failure group might be higher than the AHI that is presented, potentially influencing the outcome of 
patients with OAT failure. A logistic regression analysis was performed, and AHI did not prove to be a strong individual predictor in this patient cohort. Adjusting for the AHI, the jaw thrust maneuver proved to be a significant independent predictor.

Previously, other authors have tried to find a correlation between DISE results and OAT effectiveness. Battagel et al. and De Corso et al. have suggested that the effect of a mandibular protrusion $<5 \mathrm{~mm}$ is predictive of OAT benefit. ${ }^{12,15}$ Vanderveken et al. and Vroegop et al. have supported the concept of DISE with the addition of a simulation bite. ${ }^{3,6,23}$ Vonk et al. demonstrated that a manual jaw thrust during DISE protruding the mandible at roughly between 50 and $75 \%$ of protrusion leads to an overestimation of the effect of OAT. ${ }^{2}$ It is possible that this overestimation of the effect of OAT is present in the current study. Overestimation could account for the 13 patients in the OAT failure group with a positive jaw thrust maneuver. In a recent study by Huntley et al., the results of patients who underwent DISE and received OAT based on the recommendations during DISE were compared with a patient group who received OAT without prior selection by DISE. They found a significantly lower AHI and an increased number of patients reaching an $\mathrm{AHI}<5$ with OAT in the DISE group. ${ }^{16}$ These results are in line with the results of our study.

\section{Clinical Relevance}

To the best of our knowledge, the present study the first study to compare the results of DISE in patients with OAT failure and OAT benefit. Additionally, the present study is the first study to analyze the predictive value of the jaw thrust maneuver for the effectiveness of OAT. Without suitable predictors for failure of OAT, there is an average to large percentage of patients that is inadequately treated for a short to longer period. The findings of the present study are, therefore, of great importance for the prediction of the effectiveness of OAT. Furthermore, finding suitable predictors for selecting patients that will benefit from OAT will potentially have a beneficial effect on the cost reduction in OSA treatment. Additionally, it is expected that decreasing the group of inadequately-treated OSA patients will have a favorable effect on cost reduction in OSA healthcare in general.

\section{Limitations and Strengths}

The present study has several limitations. In the present study, the mandibular advancement maneuver was performed by manually performing a jaw thrust maneuver. Previous authors have criticized this technique, since it is nonreproducible and nontitratable and it does not account for vertical opening while closing the mouth, and state that the simulation bite is more accurate to predict the response to OAT. ${ }^{3,6,23}$ However, in daily practice, the simulation bite technique might prove to be time-consuming and costly, potentially delaying and raising the cost of adequate OSA treatment, whereas performing a jaw thrust maneuver can easily and routinely be augmented to DISE. Additionally, it has been argued that the relaxation implied by the pharma- cology necessary for DISE can possibly influence the tolerability for the jaw thrust maneuver, possibly leading to an overestimation of the OAT effect. Overestimation could possibly explain the patients in the OAT failure group with a positive jaw thrust maneuver. The assessment of the upper airway during DISE and the concomitant jaw thrust maneuver are based on subjective findings and, therefore, are prone to experience bias. Prior studies have shown DISE to be reliable and its interobserver reliability to be moderate to substantial, especially in experienced ENT surgeons. ${ }^{24-26}$ In the present study, the jaw thrust maneuver was executed by one single surgeon and was identically performed in every individual according to the description in the method section. Thus, it can be expected that the jaw thrust maneuver was very similar in each individual. With the method description, it can easily be reproduced in daily practice in other healthcare institutions. However, the fact that the jaw thrust maneuver does not exactly simulate the effect of the OAT, the difficulty of reproduction and the lack of a better system to control the sedation does affect the internal and external validity of the study. Undoubtedly, the retrospective nature of the present study is a limiting factor. The present retrospective analysis was performed in a larger research design, and currently, prospective studies are being conducted to validate the observed retrospective correlations. The present study also has several important strengths; DISE was executed by one single surgeon and the jaw thrust maneuver was performed identically in every individual. Furthermore, this is the first study to analyze the predictive value of the jaw thrust maneuver for the effectiveness of OAT.

\section{Conclusion}

According to the present retrospective analysis, a negative jaw thrust maneuver can be a valuable independent predictor for OAT failure. Therefore, we suggest that DISE should be considered as a diagnostic evaluation tool to accurately predict the success of OAT. Based on the findings of the present retrospective study, we are currently prospectively evaluating the predictive value of the jaw thrust maneuver for the effectiveness of OAT.

\section{List of abbreviations}

AHI Apnea-hypopnea index

AP Anteroposterior

AUC Area under the curve

BMI Body mass index

CPAP Continuous positive airway pressure

Co Concentric

DISE Drug-induced sleep endoscopy

ENT Ear, nose, throat

JM Jaw thrust maneuver

La Lateral

MAD Mandibular advancement device

MAM Mandibular advancement maneuver

NPV Negative predictive value

OAT Oral appliance treatment 
ODI Oxygen desaturation index

OSA Obstructive sleep apnea

PG Respiratory polygraphy

PPV Positive predictive value

PSG Polysomnography

ROC Receiver operating characteristic

VOTE Velum, oropharynx, tongue base, epiglottis

\section{Ethical Approval}

All procedures performed in studies involving human participants were in accordance with the ethical standards of the institutional and/or national research committee and with the 1964 Helsinki Declaration and its later amendments or comparable ethical standards. Data on study subjects was collected and stored anonymously to protect personal information.

\section{Manuscript Approval}

All authors declare that they have read and approved the final version of the manuscript.

\section{Availability of Data and Materials}

The dataset is available on request from: c.veugen@antoniusziekenhuis.nl

\section{Authors' Contributions}

Drug-induced sleep endoscopy was performed by Copper M P.. Data collection and analysis was done by Sanders $\mathrm{R}$. M. C. and Veugen C. C. A. F. M.. Veugen C. C. A. F. M. wrote the manuscript. Copper M P. and Stokroos R. J. provided scientific supervision. All authors read and approved the final manuscript.

\section{Funding}

No financial support received for the present research.

\section{Conflicts of Interest}

The authors have no conflict of interests to declare.

\section{Acknowledgments}

We want to thank the study participants. We thank E. Tromp for her assistance with the data analysis. J.A. Hardeman is acknowledged for critically proofreading the manuscript.

\section{References}

1 American Academy of Sleep Medicine Task Force. Sleep-related breathing disorders in adults: recommendations for syndrome definition and measurement techniques in clinical research. The Report of an American Academy of Sleep Medicine Task Force. Sleep 1999;22(05):667-689

2 Vonk PE, Beelen AMEH, de Vries N. Towards a prediction model for drug-induced sleep endoscopy as selection tool for oral appliance treatment and positional therapy in obstructive sleep apnea. Sleep Breath 2018;22(04):901-907

3 Vroegop AVMT, Vanderveken OM, Dieltjens M, et al. Sleep endoscopy with simulation bite for prediction of oral appliance treatment outcome. J Sleep Res 2013;22(03):348-355
4 Aktas O, Erdur O, Cirik AA, Kayhan FT. The role of drug-induced sleep endoscopy in surgical planning for obstructive sleep apnea syndrome. Eur Arch Otorhinolaryngol 2015;272(08): 2039-2043

5 Sullivan CE, Issa FG, Berthon-Jones M, Eves L. Reversal of obstructive sleep apnoea by continuous positive airway pressure applied through the nares. Lancet 1981;1(8225): 862-865

6 Vanderveken OM, Vroegop AV, van de Heyning PH, Braem MJ. Drug-induced sleep endoscopy completed with a simulation bite approach for the prediction of the outcome of treatment of obstructive sleep apnea with mandibular repositioning appliances. Oper Tech Otolaryngol-Head Neck Surg 2011;22(02): 175-182

7 Lindberg E, Berne C, Elmasry A, Hedner J, Janson C. CPAP treatment of a population-based sample-what are the benefits and the treatment compliance? Sleep Med 2006;7(07): 553-560

8 Ferguson KA, Cartwright R, Rogers R, Schmidt-Nowara W. Oral appliances for snoring and obstructive sleep apnea: a review. Sleep 2006;29(02):244-262

9 Sutherland K, Vanderveken OM, Tsuda H, et al. Oral appliance treatment for obstructive sleep apnea: an update. J Clin Sleep Med 2014;10(02):215-227

10 Marklund M, Stenlund H, Franklin KA. Mandibular advancement devices in 630 men and women with obstructive sleep apnea and snoring: tolerability and predictors of treatment success. Chest 2004;125(04):1270-1278

11 Croft CB, Pringle M. Sleep nasendoscopy: a technique of assessment in snoring and obstructive sleep apnoea. Clin Otolaryngol Allied Sci 1991;16(05):504-509

12 Battagel JM, Johal A, Kotecha BT. Sleep nasendoscopy as a predictor of treatment success in snorers using mandibular advancement splints. J Laryngol Otol 2005;119 (02):106-112

13 Eichler C, Sommer JU, Stuck BA, Hörmann K, Maurer JT. Does druginduced sleep endoscopy change the treatment concept of patients with snoring and obstructive sleep apnea? Sleep Breath 2013;17(01):63-68

14 Johal A, Battagel JM, Kotecha BT. Sleep nasendoscopy: a diagnostic tool for predicting treatment success with mandibular advancement splints in obstructive sleep apnoea. Eur J Orthod 2005;27 (06):607-614

15 De Corso E, Bastanza G, Della Marca GC, Rizotto G, Marchese MR, Fiorita A, Sergi B, Meucci D, Di Nardo W, Paludetti G, Scarano E. Drug-induced sleep endoscopy as a selection tool for mandibular advancement therapy by oral device. Acta Otorhinolaryngol Ital 2015;35:426-432

16 Huntley C, Cooper J, Stiles M, Grewal R, Boon M. Predicting success of oral appliance therapy in treating obstructive sleep apnea using drug-induced sleep endoscopy. J Clin Sleep Med 2018;14(08): 1333-1337

17 Kent DT, Rogers R Sr, Soose RJ. Drug-Induced Sedation Endoscopy in the Evaluation of OSA Patients with Incomplete Oral Appliance Therapy Response. Otolaryngol Head Neck Surg 2015;153(02): 302-307

18 Lechner M, Wilkins D, Kotecha B. A review on drug-induced sedation endoscopy - Technique, grading systems and controversies. Vol. 41;Sleep Medicine Reviews. W.B. Saunders Ltd; 2018: 141-148

19 Kezirian EJ, Hohenhorst W, de Vries N. Drug-induced sleep endoscopy: the VOTE classification. Eur Arch Otorhinolaryngol 2011;268(08):1233-1236

20 Op de Beeck S, Dieltjens M, Verbruggen AE, et al. Phenotypic labelling using drug-induced sleep endoscopy improves patient selection for mandibular advancement device outcome: A prospective study. J Clin Sleep Med 2019;15(08): 1089-1099 
21 Tan HL, Gozal D, Ramirez HM, Bandla HPR, Kheirandish-Gozal L. Overnight polysomnography versus respiratory polygraphy in the diagnosis of pediatric obstructive sleep apnea. Sleep (Basel) 2014; 37(02):255-260

22 Kirsch DB. PRO: sliding into home: portable sleep testing is effective for diagnosis of obstructive sleep apnea. J Clin Sleep Med 2013;9(01):5-7

23 De Vito A, Carrasco Llatas M, Ravesloot MJ, et al. European position paper on drug-induced sleep endoscopy: 2017 Update. Clin Otolaryngol 2018;43(06):1541-1552
24 Carrasco-Llatas M, Zerpa-Zerpa V, Dalmau-Galofre J. Reliability of drug-induced sedation endoscopy: interobserver agreement. Sleep Breath 2017;21(01):173-179

25 Vroegop AVMT, Vanderveken OM, Wouters K, et al. Observer variation in drug-induced sleep endoscopy: experienced versus nonexperienced ear, nose, and throat surgeons. Sleep (Basel) 2013;36(06):947-953

26 Kezirian EJ, White DP, Malhotra A, Ma W, McCulloch CE, Goldberg AN. Interrater reliability of drug-induced sleep endoscopy. Arch Otolaryngol Head Neck Surg 2010;136(04):393-397 